\title{
Microscopic calculations of double and triple giant resonance excitations in heavy ion collisions
}

\author{
E. G. Lanza and F. Catara \\ I.N.F.N.-Catania and Dipartimento di Fisica e Astronomia, Universitá di Catania, Via S. Sofia 67, I-95123 Catania, Italy \\ M. V. Andrés \\ Departamento de Física Atomica, Molecular y Nuclear, Universidad de Sevilla, Apdo 1065, E-41080 Sevilla, Spain \\ $\mathrm{Ph}$. Chomaz \\ GANIL (DSM-CEA/IN2P3-CNRS), B.P. 55027, F-14076 Caen Cédex 5, France \\ M. Fallot \\ Subatech, 4 rue Alfred Kastler BP 20722, F-44307 Nantes Cedex 3, France \\ J. A. Scarpaci \\ Institut de Physique Nucléaire, IN2P3-CNRS, F-91406 Orsay Cedex, France \\ (Received 11 October 2006; published 21 December 2006)
}

\begin{abstract}
We perform microscopic calculations of the inelastic cross sections for the double and triple excitation of giant resonances induced by heavy-ion probes within a semiclassical coupled-channels formalism. The channels are defined as eigenstates of a bosonic quartic Hamiltonian constructed in terms of collective random-phase approximation phonons. Therefore, they are superpositions of several multiphonon states, also with different numbers of phonons, and the spectrum is anharmonic. The inclusion of $(n+1)$ phonon configurations affects the states whose main component is a $n$-phonon one and leads to an appreacible lowering of their energies. We check the effects of such further anharmonicities on the previously published results for the cross section for the double excitation of giant resonances (GR). We find that the only effect is a shift of the peaks toward lower energies, the double GR cross section being unmodified by the explicity inclusion of the three-phonon channels in the dynamical calculations. The latter provide an important contribution to the cross section in the triple GR energy region, which, however, is still smaller than the experimental available data. The inclusion of four-phonon configurations in the structure calculations does not modify the results.
\end{abstract}

DOI: 10.1103/PhysRevC.74.064614 PACS number(s): 24.30.Cz, 21.60.Ev, 21.60.Jz, 25.70.De

\section{INTRODUCTION}

Since their discovery (about 70 years ago) the giant resonances (GR) have been considered the best example of coherent motion of nuclear systems [1]. From a macroscopic point of view, this collective behavior can be considered highfrequency harmonic vibrations of the nuclear density around its equilibrium shape. If these oscillations were harmonic, then higher states of equidistant energies should exist. The first experimental indication of some structures in the excitation function in heavy-ion collisions that could be interpreted as due to the population of double giant resonances dates back to 1977 (see reviews in Ref. [2] and references therein). Recently there has been unambiguous evidence of the existence of a double giant quadrupole resonance [3]. More recently the triple giant quadrupole resonance has been observed at GANIL by using, for the first time, the SPEG spectrometer in conjunction with the INDRA $4 \pi$ detector [4,5]. Multiphonon excitations were clearly observed in double charge-exchange reactions using $\left(\pi^{+}, \pi^{-}\right)$and $\left(\pi^{-}, \pi^{+}\right)$reactions [6]. The study of the excitation of the double giant dipole resonances (DGDR) of very heavy nuclei by means of relativistic Coulomb excitation have been investigated in experiments performed at GSI within the LAND Collaboration [7]. These are exclusive experiments where projectile fragments, neutron and $\gamma$ rays from the excited fragments, are measured. More recently, the same group has found a hint for a three-phonon dipole state by measuring the differential cross section for electromagnetic fission of ${ }^{238} \mathrm{U}$ at relativistic energy [8].

Theoretical studies to better understand multiphonon problems have taken various directions. An extension of the quasiparticle random-phase approximation (RPA) was used in Ref. [9], where, in addition to the mixing of one- and two-phonon states, some specific three-phonon configurations were considered as a mechanism to generate the damping width of the DGDR. Other approaches exploit the so-called Brink-Axel hypothesis [10]: a GR can be excited on top of any nuclear excited state. In this approach $[11,12]$, the states to which the one-phonon states decay are described in terms of Gaussian orthogonal ensemble (GOE). By means of the random matrix theory the average cross section for the excitation of the DGDR is calculated as a function of the spreading and damping width. The inelastic cross sections of the double and triple giant dipole resonances in a Coulomb excitation process [13] also have been calculated.

The excitation of collective vibrational states in heavy-ion collisions can be viewed as due to the action of the mean field of each collision partner on the other. A rather good microscopic description of such states is given by RPA. It can be introduced as the lowest order in a boson expansion 
leading to a boson image of the Hamiltonian that is a sum of independent harmonic oscillators corresponding to each collective mode. Thus the RPA states are pure one-phonon and multiphonon states and their energy is the sum of the energies of the single phonons. When further terms in the boson expansion are taken into account, anharmonicities arise and the eigenstates of the Hamiltonian are superpositions of multiphonon states, also with different numbers of phonons.

The mean field $U$ of each nucleus is a one-body operator. When the ground state of each nucleus is approximated by the uncorrelated Hartree-Fock (HF) one, only the particle-hole $(p h)$ part of $U$ enters. Its bosonic image is linear in the phonon creation and annihilation operators. Nonlinear terms arise in a natural way when the correlations in the ground state are taken into account and thus the particle-particle and hole-hole parts of $U$ are no more negligible. Indeed, the bosonic images of such terms are nonlinear in the RPA phonons.

In several previous articles [14-16], it has been shown that the above-mentioned anharmonicities and nonlinearities lead to a much better agreement between theoretical and experimental cross sections for the excitation of double giant resonance states in heavy-ion collisions.

Until now only the cross sections to one- and two-phonon states have been calculated within the above-mentioned approach. (For simplicity, we call the " $n$-phonon state" an eigenstate of the Hamiltonian whose main component is an $n$-phonon configuration). In Ref. [17], however, we computed the vibrational spectrum of ${ }^{40} \mathrm{Ca}$ and ${ }^{208} \mathrm{~Pb}$ by extending the previous calculations with the inclusion of three-phonon states. It was found that the two-phonon states are affected by this extension. In particular, the double GRs are appreciably pushed down. The lesson we learn from this is that one cannot perform calculations on the double GR without taking into account the effect of the three-phonon states. In view of that, in the present article we recalculate the cross section for the inelastic scattering ${ }^{40} \mathrm{Ca}+{ }^{40} \mathrm{Ca}$ at $50 \mathrm{MeV} /$ nucleon and ${ }^{208} \mathrm{~Pb}+{ }^{208} \mathrm{~Pb}$ at $641 \mathrm{MeV} /$ nucleon in the enlarged space to check how much our previous results on the cross section in the double GR region are modified. Then we present calculations of the inelastic cross sections for the triple excitation of the GR where we include the three-phonon states. In these latter calculations we also take into account the effects of the four-phonon states.

\section{APPROACHES AND FORMALISM}

In this section we briefly present our approach and how anharmonicities in the spectrum and nonlinearities in the excitation operator are treated in it. Our model makes use of standard semiclassical methods techniques. These methods are based on the assumption that nuclei move on classical trajectories, whereas the internal degrees of freedom are treated quantum mechanically.

\section{A. Multiphonon structure and anharmonicities}

Let us denote by $p(h)$ the single-particle states that are unoccupied (occupied) in the HF ground state of the nucleus and introduce the mappings [18]

$$
a_{p}^{\dagger} a_{h} \rightarrow B_{p h}^{\dagger}+(1-\sqrt{2}) \sum_{p^{\prime} h^{\prime}} B_{p^{\prime} h^{\prime}}^{\dagger} B_{p^{\prime} h}^{\dagger} B_{p h^{\prime}}+\ldots,
$$

and

$$
a_{p}^{\dagger} a_{p^{\prime}} \rightarrow \sum_{h} B_{p h}^{\dagger} B_{p^{\prime} h}^{\dagger} ; \quad a_{h}^{\dagger} a_{h^{\prime}} \rightarrow \sum_{p} B_{p h}^{\dagger} B_{p h^{\prime}}^{\dagger},
$$

where $B_{p h}^{\dagger}$ and $B_{p h}$ are bosonic operators

$$
\left[B_{p h}, B_{p^{\prime} h^{\prime}}^{\dagger}\right]=\delta_{p p^{\prime}} \delta_{h h^{\prime}} .
$$

The second term in the right-hand side of Eq. (1) is a correction taking care of the Pauli principle. The fermionic Hamiltonian is then mapped onto

$$
\begin{aligned}
H_{B}= & \left(H_{10} B^{\dagger}+H_{11} B^{\dagger} B+H_{20} B^{\dagger} B^{\dagger}\right)+\text { h.c. } \\
& +\left(H_{21} B^{\dagger} B^{\dagger} B+H_{22} B^{\dagger} B^{\dagger} B B+H_{31} B^{\dagger} B^{\dagger} B^{\dagger} B\right) \\
& + \text { h.c. },
\end{aligned}
$$

where we have dropped indices for simplicity. The term $H_{10}$ vanishes in the HF basis. Collective phonon operators are introduced by means of the Bogoliubov transformation

$$
Q_{v}^{\dagger}=\sum_{p, h}\left(X_{p h}^{v} B_{p h}^{\dagger}-Y_{p h}^{v} B_{p h}\right)
$$

The $X$ and $Y$ coefficients can be chosen so that the part of the Hamiltonian that is quadratic in the $B^{\dagger}$ and $B$ operators is diagonal when expressed in terms of the $Q^{\dagger}$ and $Q$ ones

$$
H_{\mathrm{RPA}}=\sum_{\nu} E_{v} Q_{\nu}^{\dagger} Q_{v}
$$

and the $X$ and $Y$ satisfy the RPA equations. Of course, the spectrum of $H_{\mathrm{RPA}}$ is harmonic. The other terms of the bosonic Hamiltonian (5) introduce anharmonicities because they mix multiphonon states among themselves. In our model we neglect the $H_{31}$ term because it is smaller than the others, as it has also been checked in an extended Lipkin model [19]. For the remaining terms we keep only

$$
H_{21} B^{\dagger} B^{\dagger} B+\text { h.c. } \rightarrow \mathbb{H}_{21} Q^{\dagger} Q^{\dagger} Q+\text { h.c. }
$$

and

$$
H_{22} B^{\dagger} B^{\dagger} B B \rightarrow \mathbb{H}_{22} Q^{\dagger} Q^{\dagger} Q Q,
$$

because the others are smaller by a factor $Y / X$ or powers of it. Therefore our bosonic Hamiltonian becomes

$$
\begin{aligned}
\mathbb{H}_{Q}= & \sum_{\nu} E_{\nu} Q_{\nu}^{\dagger} Q_{\nu}+\sum_{\nu_{1} \nu_{2} v} \mathbb{H}_{21} Q_{\nu_{1}}^{\dagger} Q_{\nu_{2}}^{\dagger} Q_{\nu}+\text { h.c. } \\
& +\sum_{v_{1} \nu_{2} v_{1}^{\prime} v_{2}^{\prime}} \mathbb{H}_{22} Q_{\nu_{1}}^{\dagger} Q_{\nu_{2}}^{\dagger} Q_{v_{1}^{\prime}} Q_{v_{2}^{\prime}} .
\end{aligned}
$$

The eigenstates and eigenvalues of $\mathbb{H}_{Q}$ are then found by diagonalizing it in the space of the states containing up to a certain number $N_{\text {pho }}$ of phonons. The $\mathbb{H}_{22}$ term mixes multiphonon states with the same number of phonons, whereas the $\mathbb{H}_{21}$ mixes states having number of phonons differing by one. In Ref. [17] we showed the results for ${ }^{40} \mathrm{Ca}$ and ${ }^{208} \mathrm{~Pb}$ obtained in the space with $N_{\text {pho }}=3$ and we compared them 
with those of Ref. [15], where we had $N_{\text {pho }}=2$. In the next section we mention the main results obtained there.

\section{B. Nonlinearities in the excitation operator}

Within a semiclassical approach to nucleus-nucleus collisions the excitation of one partner (say A) is due to the mean field of the other (B) acting on it. Therefore the excitation operator is

$$
W(t)=\sum_{i j} W_{i j}(t) a_{i}^{\dagger} a_{j}
$$

where $W_{i j}(t)=\left(i\left|U_{B}(\vec{R}(t))\right| j\right)$ and $U_{B}$ is the mean field of $B$ (including Coulomb), which depends on time through the relative distance between the two nuclei. The indices $i$ and $j$ denote single-particle states, both occupied and unoccupied, in nucleus A. When one neglects the correlation present in the ground state, only the $p h$ terms of $W$ are effective and the boson image of $W$ is linear in the $Q^{\dagger}$ and $Q$ operators. Taking into account the correlations the $p p$ and $h h$ parts of $W$ cannot be neglected and this brings to quadratic terms

$$
\begin{aligned}
W= & W^{00}+\left(\sum_{v} W_{v}^{10} Q_{v}^{\dagger}+\sum_{\nu v^{\prime}} W_{v v^{\prime}}^{11} Q_{v}^{\dagger} Q_{v^{\prime}}\right. \\
& \left.+\sum_{v v^{\prime}} W_{\nu v^{\prime}}^{20} Q_{v}^{\dagger} Q_{v^{\prime}}^{\dagger}\right)+ \text { h.c. }
\end{aligned}
$$

The first term in this equation represents the interaction of the two colliding nuclei in their ground state. The $W^{10}$ part connects states differing by one phonon, the $W^{11}$ term couples excited states with the same number of phonons, whereas $W^{20}$ allows coupling between states differing by two phonons. These new routes of excitation may increase the excitation probability of the multiple GR.

\section{The cross section}

The inelastic scattering cross section is calculated within a semiclassical coupled-channels approach. Let $\left|\Phi_{\alpha}\right\rangle$ denote the excited state of the nucleus of which we want to calculate the excitation probability. These states are eigenstates of the Hamiltonian (9) and therefore are superpositions of multiphonon states obtained by diagonalizing $\mathbb{H}_{Q}$ in the space containing up to $N_{\text {pho }}$ phonon configurations. The excitation probability amplitudes satisfy, for each impact parameter $b$, the set of coupled differential equations

$$
\dot{A}_{\alpha}(t)=-i \sum_{\alpha^{\prime}} e^{i\left(E_{\alpha}-E_{\alpha^{\prime}}\right) t}\left\langle\Phi_{\alpha}|W(t)| \Phi_{\alpha^{\prime}}\right\rangle A_{\alpha^{\prime}}(t),
$$

which are integrated along classical trajectories with various impact parameters $b[15,16]$. The cross section to excite the state $\left|\Phi_{\alpha}\right\rangle$ is then calculated as

$$
\sigma_{\alpha}=2 \pi \int_{0}^{+\infty} P_{\alpha}(b) T(b) b d b,
$$

where $P_{\alpha}(b)=\left|A_{\alpha}(b, t=+\infty)\right|^{2}$. The integral is over the whole impact parameters range that is modulated by the transmission coefficient $T(b)$.

\section{EXCITATION OF ${ }^{40} \mathrm{CA}$}

In this section we briefly recall the results we obtained for the structure calculation for the ${ }^{40} \mathrm{Ca}$ [17]. As stated also in the Introduction, calculations were guided by the results we got from a study of an extended Lipkin-Meshow-Glick (LMG) model [19]. In that article, the original LMG model has been extended to include terms that play the same role than the anharmonic terms of our Hamiltonian (9). The Hamiltonian of such an extended LMG model is still exactly solvable. The relevant results can be summarized as follows: its diagonalization in an enlarged space, including up to three-phonon states, produces results that are very close to the exact ones [19]. Therefore we have followed this approach to calculate the spectrum of ${ }^{40} \mathrm{Ca}$ in the space of one-, two-, and three-phonon states.

We used a discrete self-consistent HF+RPA with a SGII interaction, including all one-phonon states with $J \leqslant 3$ that exhaust at least $5 \%$ of the energy-weighted sum rule. For the nucleus ${ }^{40} \mathrm{Ca}$, we used the nine one-phonon basis shown in Table I. We constructed all two- and three-phonon configurations out of them, without energy cutoff, with both natural and unnatural parity. Then the Hamiltonian (9) has been diagonalized in the space spanned by such states. The eigenstates are mixed states whose components are of one-, two-, and three-phonon kind:

$$
\left|\Phi_{\alpha}\right\rangle=\sum_{v_{1}} c_{\nu_{1}}^{\alpha}\left|\nu_{1}\right\rangle+\sum_{\nu_{1} \nu_{2}} c_{v_{1} \nu_{2}}^{\alpha}\left|v_{1} \nu_{2}\right\rangle+\sum_{\nu_{1} \nu_{2} \nu_{3}} c_{\nu_{1} \nu_{2} \nu_{3}}^{\alpha}\left|\nu_{1} \nu_{2} \nu_{3}\right\rangle
$$

The inclusion of the three-phonon states changes the energies of the phonon basis of a few hundred of $\mathrm{keV}$ as shown in Table I. The main result of the calculation is that the spectrum of the two-phonon states is strongly modified by their coupling to the three-phonon ones. The diagonalization in the three-phonon space produces very large shifts in the energies,

TABLE I. RPA one-phonon basis for the nucleus ${ }^{40} \mathrm{Ca}$. For each state, spin, parity, energy, and percentage of the EWSR(isovector for the GDR and the IVGQR and isoscalar for all the other states) are reported. In the last two columns we report the energies of the phonons after the inclusion of two- and three-phonon states, respectively.

\begin{tabular}{llcccc}
\hline \hline State & $J^{\pi}$ & $E_{\text {harm }}(\mathrm{MeV})$ & $\operatorname{EWSR}(\%)$ & $E_{2 p h}(\mathrm{MeV})$ & $E_{3 p h}(\mathrm{MeV})$ \\
\hline $\mathrm{GMR}_{1}$ & $0^{+}$ & 18.25 & 30 & 18.36 & 18.30 \\
$\mathrm{GMR}_{2}$ & $0^{+}$ & 22.47 & 54 & 22.00 & 21.78 \\
$\mathrm{GDR}_{1}$ & $1^{-}$ & 17.78 & 56 & 17.35 & 17.29 \\
$\mathrm{GDR}_{2}$ & $1^{-}$ & 22.03 & 10 & 21.64 & 21.59 \\
$\mathrm{ISGQR}$ & $2^{+}$ & 16.91 & 85 & 16.51 & 16.44 \\
$\mathrm{IVGQR}$ & $2^{+}$ & 29.59 & 26 & 29.09 & 29.00 \\
$3^{-}$ & $3^{-}$ & 4.94 & 14 & 4.47 & 4.40 \\
LEOR & $3^{-}$ & 9.71 & 5 & 9.33 & 9.28 \\
HEOR & $3^{-}$ & 31.33 & 25 & 30.80 & 30.89 \\
\hline \hline
\end{tabular}


more than $1 \mathrm{MeV}$ (for ${ }^{40} \mathrm{Ca}$ ) in almost all the cases and always downward. In view of these results, the inelastic scattering cross section to two-phonon states has to be recalculated and compared with that obtained without the inclusion of three-phonon configurations [16].

\section{A. Cross section at $50 \mathrm{MeV} /$ nucleon}

The semiclassical calculations for the reaction ${ }^{40} \mathrm{Ca}+{ }^{40} \mathrm{Ca}$ at $50 \mathrm{MeV} /$ nucleon have been performed within the framework described above. In this section we discuss the inelastic crosssection calculations done by including up to the three-phonon states.

In the case we are interested in, the nuclear contribution is important. The form factors have been calculated [20] by employing a double folding procedure with the transition densities calculated within the RPA. Furthermore, it has been introduced an optical potential to avoid the uncertainty on the integration over the impact parameters. Because the optical potential takes into account the absorption due to all channels, we have introduced a procedure to avoid double counting the effects of the channels explicitly included in our calculations [16].

The number of two- and three-phonon states constructed from the one-phonon basis given in Table $\mathrm{I}$ is more than 1,000. Considering that the amplitudes are complex quantities and that we have an equation for each angular momentum and its projection, the number of time-dependent coupled equations to solve amounts to about 10,000 . We have then to reduce their number to render the calculation feasible. We took into account only the natural-parity states and furthermore we have considered for the calculations only states with an excitation energy below $60 \mathrm{MeV}$. This cutoff in the excitation energies guarantees that we take into account almost all the two-phonon states and a great number of the three-phonon ones. Furthermore, we took into account, for each state, only the components whose value is larger than 0.03 . This choice guaranties still a very good normalization and reduces appreciably the computation time.

The contribution of the three-phonon states to the inelastic cross section for ${ }^{40} \mathrm{Ca}+{ }^{40} \mathrm{Ca}$ at $50 \mathrm{MeV} /$ nucleon can be appreciated in Fig. 1, where the results of the calculations in the larger space (solid line) are compared with the twophonon ones (dot-dashed line) [16]. Actually, we calculated the inelastic cross section for each individual state (14) by solving the coupled-channels equations (12). The curves presented here are always the result of a smoothing procedure with a Lorentzian with a width of $\Gamma=5 \mathrm{MeV}$ ( $7 \mathrm{MeV}$ for excitation energies greater than $30 \mathrm{MeV}$ ) of the theoretical cross sections to the discrete levels. It appears that both the one-phonon and the two-phonon strengths are influenced a bit by the inclusion of the three-phonon states. Although the threephonon configurations appreciably affect both the energies and wave functions of the two-phonon states [17], their role in the calculation of the cross section seems to be small. On the contrary, in the three-phonon region the increase of the cross section is appreciable and one can see that the inclusion of the three-phonon components improves the agreement with

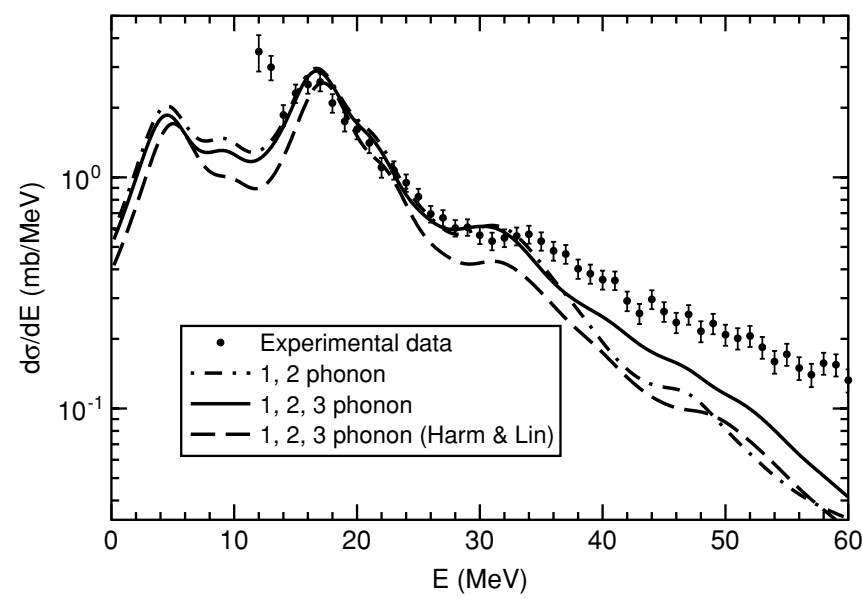

FIG. 1. Comparison of the cross section for ${ }^{40} \mathrm{Ca}+{ }^{40} \mathrm{Ca}$ at $50 \mathrm{MeV} /$ nucleon computed, including only one and two phonons (dot-dashed line) with the complete calculation, namely with one, two, and three phonons (solid line). The dashed line correspond to the complete calculation in the harmonic and linear case. For the experimental data see Ref. [16].

the experimental data although there is still some cross section missing. One might argue that a further enlargement of the diagonalization space by including up to four-phonon states could reduce this discrepancy. As shown in the next section this is not the case. Therefore some processes that are not taken into account in our approach might be present in this energy region. Indeed, the experimental spectrum compared with the calculations is an excitation energy spectrum of ${ }^{40} \mathrm{Ca}$ in coincidence with only one detected proton at backward angles. This coincidence measurement makes sure that below $35 \mathrm{MeV}$ no reaction mechanism participates to the inelastic channel. However, above this excitation energy, a second particle can be emitted in the forward direction through a different reaction mechanism (such as the towing mode), leading to a higher cross section than for a simple excitation. This can be evidenced by means of velocity plots [21] that clearly show an asymmetry. Even though one proton is emitted backward another one can be emitted forward contributing to an increased cross section around $40 \mathrm{MeV}$.

In Fig. 1 it is also plotted a curve (dashed) that corresponds to the complete calculation in the harmonic and linear case. Here, once again, we underline the importance of the anharmonic and nonlinear contributions. Indeed, from the figure one infers that their presence increases the cross section along the whole energy range of the calculation, expecially in the double and triple GR energy regions. The increase of the cross section in the triple GR energy region could be thought to be due only to the presence of many more states in that region when three-phonon states are included. The comparison with the results in the harmonic and linear limit, where we have the same number of states, puts in evidence the real origin of the strong increase. Namely, as already stressed in Refs. $[15,16,20]$, the nonlinearities open new routes to the excitation of multiphonon states, whereas the anharmonicities allow to populate them through their one- and/or two-phonon components. 


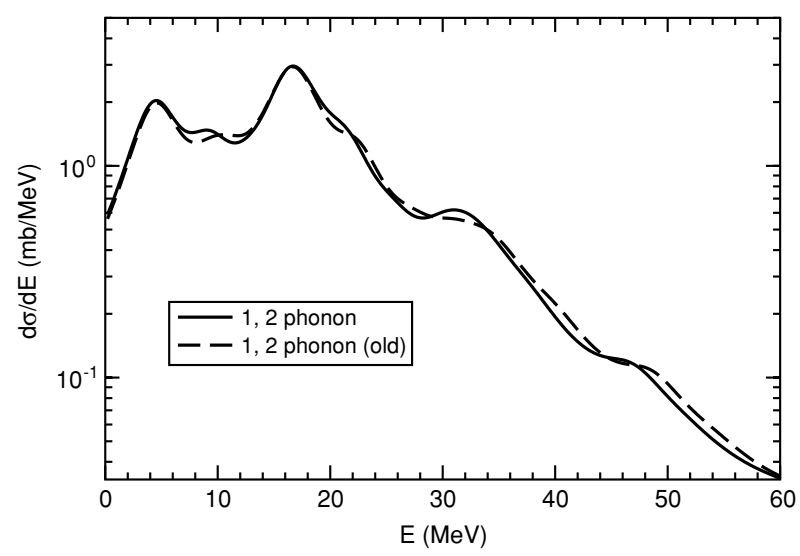

FIG. 2. Comparison of the inelastic cross section for ${ }^{40} \mathrm{Ca}+{ }^{40} \mathrm{Ca}$ at $50 \mathrm{MeV} /$ nucleon between a calculation, including only one- and two-phonon states, but with the energies and wave functions coming from the diagonalization in the space of up to three-phonon states (solid line) and the old two-phonon calculations (dashed line).

In Fig. 2 we compare the old two-phonon calculation of Ref. [16] with the one done including only one- and twophonon channels but with the energies and wave functions coming from the diagonalization of the Hamiltonian (9) in the space of up to three-phonon states. We note that the different calculations produce very similar results. The single resonance peak is exactly the same in energy, intensity, and shape, whereas a bit of a difference can be noted in the two-phonon region as well as in the high-energy region. The main effect, in these regions, is a small shift toward lower energies of the whole curve, which is due to the relatively strong anharmonicities we found when the three-phonon states were included in the diagonalization. In any case, the two curves are almost indistinguishable also in comparison with the experimental data.

This study shows that the calculation of the cross section can be considered to be converged at the $n$-phonon level when the $(n+1)$-phonon states are taken into account in the structure calculation but are neglected in the dynamical excitation. In the next section we apply this recipe to the three-phonon excitation, i.e., we include the four-phonon configurations in the structure but not in the coupled-channels calculations.

To get a deeper insight in the role of the three-phonon states, in Fig. 3 we have decomposed the inelastic cross section into the one-, two-, and three-phonon components. The one-phonon distribution is dominated by the low-lying states and the giant quadrupole resonance. The small peak at around $30 \mathrm{MeV}$ corresponds to the excitation of the high-energy octopole resonances (HEOR) state. All these states are excited dominantly at low impact parameters through the nuclear interaction. The incident energy and the projectile charge are not large enough to induce a strong Coulomb excitation, so the GDR cross section is four times smaller than the giant quadrupole resonance (GQR) one. The two-phonon contribution appears to be rather strong, about one order of magnitude lower than the single-phonon component. Its structure is more complex, the various bumps being related to the double low-lying state excitation, the excitation of a GQR

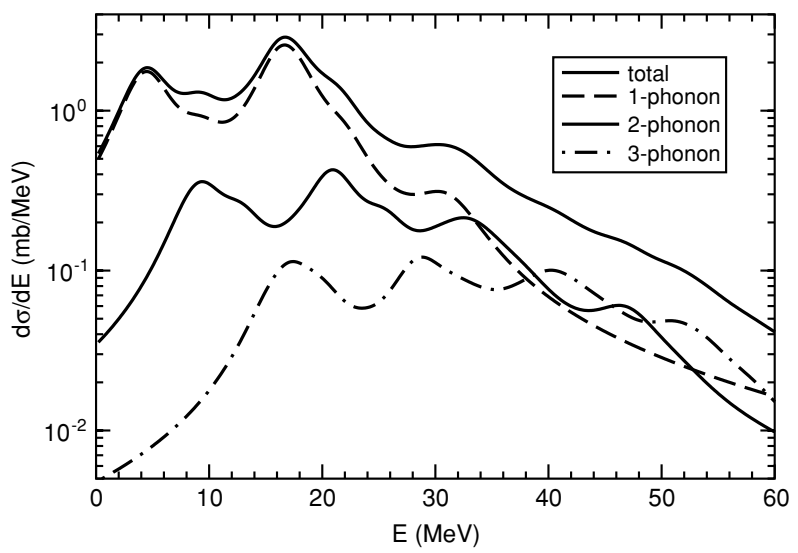

FIG. 3. Decomposition of the total inelastic cross section (solid line, upper one) for ${ }^{40} \mathrm{Ca}+{ }^{40} \mathrm{Ca}$ at $50 \mathrm{MeV} /$ nucleon into the one(dashed line), two- (solid, lower one), and three-phonon components (dot-dashed line).

on top of the low-lying mode, the double GQR, and the $L=$ 5 component of the $|\mathrm{GQR} \times \mathrm{HEOR}\rangle$ state in the high-energy tail. The latter contribution is also visible in Fig. 4. The double GQR clearly dominates the inelastic spectrum around $35 \mathrm{MeV}$ excitation energy.

The three-phonon component appears to be important. It is smaller than the two-phonon strength by a factor of about 3 . In the high-energy part it becomes the dominant contribution with a structure due to the excitation of a low-lying mode on top of the double GQR state and above $50 \mathrm{MeV}$ to the triple GQR phonon. By inspection one can infer the difference from the old calculation where only one- and two-phonon states were taken into account. In Table II we show the summed cross sections in the three indicated regions, around the energies corresponding to the excitation of one, two, and three GQR phonons, respectively. One can see that the cross sections

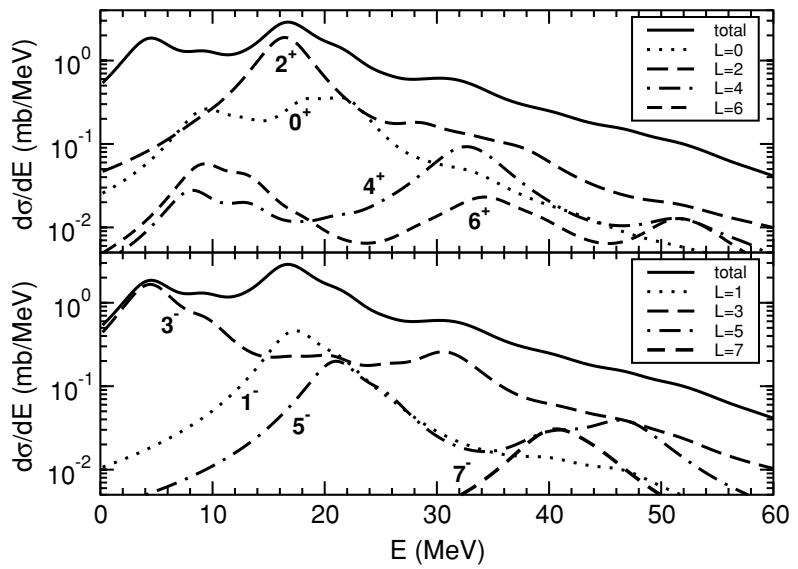

FIG. 4. Decomposition of the total inelastic cross section (solid line, in both parts) for ${ }^{40} \mathrm{Ca}+{ }^{40} \mathrm{Ca}$ at $50 \mathrm{MeV} /$ nucleon into different angular momenta contributions. The figure is divided for reader convenience. In the upper part we show the even natural-parity ones: $L=0$ (dotted), $L=2$ (long-dashed), $L=4$ (dot-dashed), and $L=$ 6 (short-dashed). In the lower part we plot the odd natural-parity angular momenta contribution: $L=1$ (dotted), $L=3$ (long-dashed), $L=5$ (dot-dashed), and $L=7$ (short-dashed). 
TABLE II. Integrated cross section (in mb) for ${ }^{40} \mathrm{Ca}+{ }^{40} \mathrm{Ca}$ at $50 \mathrm{MeV} /$ nucleon in different energy bins corresponding to one-, two-, and three-phonon regions, respectively. Shown in the first row are the results for the three-phonon complete calculations. Shown in the second row are the results corresponding to the calculations done including only one- and two-phonon states. In parenthesis the values corresponding to the single, double, and triple GQR states.

\begin{tabular}{llcl}
\hline \hline & $(14-20 \mathrm{MeV})$ & $(28-38 \mathrm{MeV})$ & $(38-60 \mathrm{MeV})$ \\
\hline Three phonons & $20.83(14.71)$ & $5.44(1.34)$ & $2.02(0.20)$ \\
Two phonons & $22.80(15.62)$ & $5.48(1.97)$ & $0.66(-)$ \\
\hline \hline
\end{tabular}

corresponding to the pure GQR states (within parentheses) decrease by a factor of about 10 each time a new phonon is excited. For comparison, we show also, in the second row, the results corresponding to the calculations done with only oneand two-phonon states.

Because of the complex structure of both the two- and threephonon strength the total cross section appears rather smooth above the double GQR bump. However, the large cross section makes possible hunting for multiphonon states using selective signals such as specific decays or multipolar decomposition.

The decomposition of the inelastic cross section into various multipoles is presented in Fig. 4, where we show the most significant contributions. To avoid an unreadable figure, full of lines, we have separated it in two parts. In the upper one, we show the even natural-parity multipoles contributions, whereas in the lower part we plot the odd natural-parity ones. The total cross section is plotted in both graphs. The contribution corresponding to the angular momenta $L=0$ and $L=1$ present appreciable peaks only in correspondence of the single GMR and GDR, with a contribution of the double $3^{-}(L=$ 0 component) for the monopole case. The single $3^{-}$collective state dominates the low-energy region. Comparing this figure with the multiphonon decomposition one can deduce that the bump in the $3^{-}$strength at high energy, around $30 \mathrm{MeV}$, i.e., in the DGR region, is due to the HEOR state. The shoulder at higher energy is a mixing of two- and three-phonon states. The quadrupole strength presents a strong peak due to the GQR. The higher energy structures are mainly due to the DGQR state except at very high energy, above $50 \mathrm{MeV}$, which is dominated by the three-GQR multiplet as can be inferred by the presence of the same structure in the $2^{+}, 4^{+}$, and $6^{+}$components. The strong peak in the $L=5$ contribution is due to the double phonon state $\left|\mathrm{GQR} \times 3^{-}\right\rangle$, whereas the one at higher energy correspond to the excitation of the $|\mathrm{GQR} \times \mathrm{HEOR}\rangle$ state. The $7^{-}$strength is due to the double GQR build on top of the low-lying $3^{-}$state.

Because the presented strength is built from the monopole, dipole, quadrupole and octupole collective states, the threephonon component goes up to $L=9$. However, it appears that the angular momenta above $L=7$, which require threephonon excitations, are a minor contribution to the total strength.

\section{B. Role of four-phonons}

The results from the previous calculation of anharmonicities evaluated in a basis including up to three-phonon states give us a clear indication: it is not possible to compute the energies of three-phonon states without including four-phonon states in the basis. This extension of the basis should be sufficient if we consider the weak influence of the three-phonon states on the one-phonon ones. Moreover, the results are well reproduced by second -order perturbation theory. The introduction of four-phonon states in the calculation could allow testing of the convergence of the series, and to study their effects on one- and two-phonon states. Indeed, the threephonon states would undergo a strong energy shift toward low energies, and their influence on two-phonon states could be substantially modified. We extend our basis to four-phonon states to compute anharmonicities and we will look at the effects on the three-phonon states.

To do that, we follow the same approach described before: The quartic Hamiltonian matrix (9) is diagonalized in the space of up to four-phonon states, thus obtaining the new mixed eigenvectors:

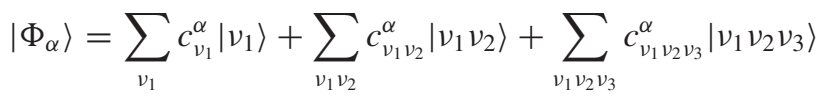

$$
\begin{aligned}
& +\sum_{v_{1} v_{2} v_{3} v_{4}} c_{v_{1} v_{2} v_{3} v_{4}}^{\alpha}\left|v_{1} v_{2} v_{3} v_{4}\right\rangle
\end{aligned}
$$

In Table III we report the results of this calculation for some relevant two-phonon states. Looking at the new energies, in the sixth column, we remark that the additional shift imparted to

TABLE III. Results of the diagonalization for some two-phonon states of ${ }^{40} \mathrm{Ca}$. In the first column, the states are labeled by their main component in the eigenvector and the corresponding unperturbed energy, indicated in parentheses. The second column indicates the amplitude of the main component $c_{0}$. Then for each total angular momentum, we show the results of the calculation in the basis up to two phonon states, the results for the basis extended to three phonon states, and the results for the basis up to four phonon states. The last column contains the results in second-order perturbation theory. All energies are given in $\mathrm{MeV}$.

\begin{tabular}{lrlrrrr}
\hline \hline $\begin{array}{l}\text { Main } \\
\text { component }\end{array}$ & \multicolumn{1}{c}{$c_{0}$} & $J^{\pi}$ & $\leqslant 2 p h$ & $\leqslant 3 p h$ & $\leqslant 4 p h$ & \multicolumn{1}{l}{$\begin{array}{l}\text { 2nd } \\
\text { order }\end{array}$} \\
\hline $3^{-} \otimes 3^{-}$ & -0.91 & $0^{+}$ & 10.96 & 9.27 & 8.77 & 9.20 \\
$(9.88)$ & -0.96 & $2^{+}$ & 10.63 & 8.89 & 8.43 & 8.75 \\
& -0.96 & $4^{+}$ & 9.85 & 8.10 & 7.64 & 7.96 \\
& -0.96 & $6^{+}$ & 10.88 & 9.12 & 8.67 & 8.99 \\
$D_{1} \otimes D_{1}$ & -0.92 & $0^{+}$ & 35.27 & 33.71 & 33.35 & 33.59 \\
$(35.56)$ & -0.96 & $2^{+}$ & 35.10 & 33.66 & 33.33 & 33.59 \\
$D_{1} \otimes Q_{1}$ & 0.95 & $1^{-}$ & 34.83 & 33.35 & 33.05 & 33.24 \\
$(34.69)$ & 0.96 & $2^{-}$ & 34.56 & 33.22 & 32.92 & 33.16 \\
& -0.96 & $3^{-}$ & 34.67 & 33.13 & 32.82 & 33.02 \\
$Q_{1} \otimes Q_{1}$ & -0.87 & $0^{+}$ & 33.88 & 32.47 & 32.03 & 32.27 \\
$(33.82)$ & 0.84 & $2^{+}$ & 33.82 & 32.47 & 32.01 & 32.26 \\
& 0.90 & $4^{+}$ & 34.02 & 32.61 & 32.18 & 32.44 \\
$M_{2} \otimes D_{1}$ & -0.89 & $1^{-}$ & 40.26 & 38.14 & 37.72 & 37.65 \\
$(40.25)$ & & & & & & \\
$M_{2} \otimes Q_{1}$ & -0.73 & $2^{+}$ & 39.62 & 37.34 & 36.50 & 36.80 \\
$(39.38)$ & & & & & & \\
$M_{2} \otimes M_{2}$ & 0.67 & $0^{+}$ & 45.60 & 42.76 & 41.15 & 41.18 \\
$(44.94)$ & & & & & & \\
\hline \hline
\end{tabular}


TABLE IV. Results of the diagonalization in the space including up to four-phonon states. The first column contains the name of the main component of the eigenvector. The harmonic energy of this state is written below the name. We show also the value of the $c$ coefficient of the main component (second column) of the state as well as its parity and total angular momentum (third column). In the fourth and fifth columns we show the eigenenergies obtained when the diagonalization is done in a space up to three and four phonons, respectively. These results have to be compared with the result of a second-order perturbation theory calculation (sixth column). Finally, in the last two columns, other important components and the corresponding $c$ coefficient are shown.

\begin{tabular}{|c|c|c|c|c|c|c|c|}
\hline Main component & $c_{0}$ & $J^{\pi}$ & $\leqslant 3 p h$ & $\leqslant 4 p h$ & 2 nd order & Important components & $c_{i}$ \\
\hline $\begin{array}{l}M_{1} \otimes M_{1} \otimes M_{1} \\
54.74\end{array}$ & -0.499 & $0^{+}$ & 54.48 & 53.12 & 50.47 & $\begin{array}{c}M_{1} \otimes 3^{-} \otimes 3^{-} \\
M_{1} \otimes 3^{-} \otimes O_{1} \\
M_{1} \otimes M_{1} \otimes M_{2} \\
M_{1} \otimes M_{1} \otimes M_{1} \otimes M_{1}\end{array}$ & $\begin{array}{r}-0.42 \\
0.41 \\
0.26 \\
0.22\end{array}$ \\
\hline $\begin{array}{l}\left(D_{1} \otimes D_{2}\right)_{2} \otimes Q_{1} \\
56.72\end{array}$ & -0.37 & $2^{+}$ & 56.17 & 53.37 & 53.09 & $\begin{array}{c}\left(D_{1} \otimes D_{2}\right)_{1} \otimes Q_{1} \\
\left(D_{1} \otimes Q_{2}\right)_{2} \otimes O_{1} \\
\left(D_{1} \otimes D_{2}\right)_{2} \otimes\left(3^{-} \otimes O_{1}\right)_{2} \\
\left(3^{-} \otimes 3^{-}\right)_{2} \otimes\left(M_{2} \otimes Q_{1}\right)_{2} \\
\left(Q_{1} \otimes Q_{1}\right)_{0} \otimes\left(O_{1} \otimes O_{1}\right)_{2} \\
\left(Q_{1} \otimes Q_{1}\right)_{2} \otimes\left(O_{1} \otimes O_{1}\right)_{0} \\
\left(Q_{1} \otimes Q_{1}\right)_{4} \otimes\left(O_{1} \otimes O_{1}\right)_{2} \\
\left(Q_{1} \otimes Q_{1}\right)_{2} \otimes\left(O_{1} \otimes O_{1}\right)_{4} \\
\left(Q_{1} \otimes Q_{1}\right)_{2} \otimes\left(O_{1} \otimes O_{1}\right)_{2}\end{array}$ & $\begin{array}{r}0.32 \\
-0.19 \\
-0.21 \\
0.13 \\
0.36 \\
0.34 \\
0.36 \\
0.31 \\
-0.20\end{array}$ \\
\hline $\begin{array}{l}Q_{1} \otimes Q_{1} \otimes Q_{1} \\
50.73\end{array}$ & -0.91 & $0^{+}$ & 50.74 & 47.8 & 47.3 & $\begin{array}{c}\left(Q_{1} \otimes Q_{1}\right)_{2} \otimes\left(3^{-} \otimes 3^{-}\right)_{2} \\
Q_{1} \otimes Q_{1} \otimes Q_{1} \otimes M_{2}\end{array}$ & $\begin{array}{r}0.24 \\
-0.27\end{array}$ \\
\hline $\begin{array}{l}Q_{1} \otimes Q_{1} \otimes Q_{1} \\
50.73\end{array}$ & -0.91 & $2^{+}$ & 50.96 & 48.0 & 47.5 & $\begin{array}{c}\left(Q_{1} \otimes Q_{1}\right)_{0} \otimes\left(3^{-} \otimes 3^{-}\right)_{2} \\
Q_{1} \otimes Q_{1} \otimes Q_{1} \otimes M_{2} \\
Q_{1} \otimes Q_{1} \otimes Q_{1} \otimes M_{1}\end{array}$ & $\begin{array}{l}-0.14 \\
-0.27 \\
-0.17\end{array}$ \\
\hline $\begin{array}{l}Q_{1} \otimes Q_{1} \otimes Q_{1} \\
50.73\end{array}$ & -0.65 & $4^{+}$ & 51.02 & 48.0 & 47.56 & $\begin{array}{c}3^{-} \otimes 3^{-} \otimes 3^{-} \otimes O_{2} \\
Q_{1} \otimes Q_{1} \otimes Q_{1} \otimes M_{2} \\
Q_{1} \otimes Q_{1} \otimes Q_{1} \otimes M_{1} \\
\left(Q_{1} \otimes Q_{1}\right)_{4} \otimes\left(3^{-} \otimes 3^{-}\right)_{2} \\
M_{2} \otimes D_{1} \otimes\left(Q_{2} \otimes 3^{-}\right)_{3} \\
M_{1} \otimes D_{1} \otimes\left(Q_{2} \otimes 3^{-}\right)_{3}\end{array}$ & $\begin{array}{r}-0.18 \\
-0.19 \\
-0.13 \\
-0.12 \\
0.18 \\
0.14\end{array}$ \\
\hline $\begin{array}{l}Q_{1} \otimes Q_{1} \otimes Q_{1} \\
50.73\end{array}$ & -0.92 & $6^{+}$ & 51.34 & 48.3 & 47.85 & $\begin{array}{c}Q_{1} \otimes Q_{1} \otimes Q_{1} \otimes M_{2} \\
\left(Q_{1} \otimes Q_{1}\right)_{4} \otimes\left(3^{-} \otimes 3^{-}\right)_{2} \\
Q_{1} \otimes Q_{1} \otimes Q_{1} \otimes M_{1}\end{array}$ & $\begin{array}{l}-0.27 \\
-0.20 \\
-0.18\end{array}$ \\
\hline
\end{tabular}

the two-phonon states by the inclusion of the four-phonon states is smaller than that in the previous case. Moreover, the new shift is always toward lower energies.

The characteristics of a few three-phonon states computed in the new basis are presented in Table IV. Anharmonicities of three-phonon states are still well reproduced by second -order perturbation theory and the correction to the harmonic energy is still negative. The energy shift is due to the presence of the four-phonon states that push downward the three-phonon ones. This can be understood looking at the second-order correction to the energy in perturbative theory, where the ratio between the matrix elements of the residual interaction appearing at the numerator and the difference in energy at the denominator determine the properties of the state. The largest matrix elements are those coupling three-phonon states to four-phonon ones. This is especially true in the cases involving triple and quadruple GMR states and arises from symmetry properties of the phonons, obeying the Bose statistics. In general, similarly to the findings of Ref. [17], the matrix elements connecting a $n$-phonon state to that formed by adding to it a GMR are large. The sign of the correction to the energy comes from the denominator, i.e., the difference between the energy of the considered state and the four-phonon states, the latter being nearly all located at higher energies.

The presence of the four-phonon states in the diagonalization basis generates eigenfunctions that are more mixed than the ones of the previuos calculations. So we get states having a main component of the order of 0.5 and several others almost as large as it. Some examples are given in Table IV. The extreme case is the $2+$ state at $53.37 \mathrm{MeV}$ excitation energy, whose main component is $\left(D_{1} \otimes D_{2}\right)_{2} \otimes Q_{1}$ and appears with an amplitude of -0.37 in the wave function. This state has several other components as large as that, which are made of three and four phonons.

The same is true for the $L=4$ state whose main component is $Q_{1} \otimes Q_{1} \otimes Q_{1}$ with an amplitude of -0.65 . The same state is much less mixed when the diagonalization is done in the space up to three-phonon states. In the latter case its 
main coefficient is 0.986 with only one big component corresponding to the state $\left(D_{1} \otimes D_{2}\right)_{2} \otimes Q_{1}$ whose amplitude is $c=0.15$. Another interesting result, found in Ref. [17] and related to the strong coupling regime, is the existence of some states having as second large component a configuration that is not directly coupled to the main one by the residual interaction. This is a second-order effect due to the fact that both these configurations have large matrix element with another one.

To check the stability of the results on the inelastic scattering cross sections to two- and three-phonon states, we have repeated the calculations by using the energies and wave functions obtained by diagonalizing the Hamiltonian in the large space but not including the four-phonon channels. We do not show the results of this calculations because they are almost indistinguishable from the previous ones. Once again, the inclusion of the $n+1$ phonons is very important in the structure of the $n$-phonons but it seems it does not affect strongly the dynamics. Therefore we can conclude that convergence has been reached (at least numerically) and the discrepancy between theory and experiment in the three-phonon region is due to the presence in the experimental data of some processes that are not taken into account in our approach.

\section{EXCITATION OF ${ }^{208} \mathrm{~Pb}$}

We have also performed calculations for multiple excitation of ${ }^{208} \mathrm{~Pb}$. The inelastic cross section for the system ${ }^{208} \mathrm{~Pb}+{ }^{208} \mathrm{~Pb}$ has been computed for an incident energy of $641 \mathrm{MeV} /$ nucleon. At this energy the nuclear contribution is believed to be small, so only the relativistic Coulomb excitation has been taken into account in the same way as it is described in Ref. [15]. The collective RPA basis states considered in the present calculation are listed in Table V. As in the previous case we construct all the possible two- and three-phonon states and we diagonalize the Hamiltonian in this space. For this case we do not take into account the effects of the four-phonon states.

In Fig. 5 we compare the complete calculation going up to three- phonons (solid line) with the previously published [15] inelastic cross section for ${ }^{208} \mathrm{~Pb}+{ }^{208} \mathrm{~Pb}$ at $641 \mathrm{MeV} /$ nucleon where only one- and two-phonon states were included. One can see that below $35 \mathrm{MeV}$ the results are not affected by the inclusion of three-phonon states. Only a small reduction of the peak around $25 \mathrm{MeV}$, corresponding to the double GDR,

TABLE V. Same as in Table I for ${ }^{208} \mathrm{~Pb}$.

\begin{tabular}{llcccc}
\hline \hline State & $J^{\pi}$ & $E_{\text {harm }}(\mathrm{MeV})$ & $\operatorname{EWSR}(\%)$ & $E_{2 p h}(\mathrm{MeV})$ & $E_{3 p h}(\mathrm{MeV})$ \\
\hline $\mathrm{GMR}_{1}$ & $0^{+}$ & 13.61 & 61 & 13.42 & 13.48 \\
$\mathrm{GMR}_{2}$ & $0^{+}$ & 15.02 & 28 & 14.78 & 14.76 \\
$\mathrm{GDR}_{1}$ & $1^{-}$ & 12.43 & 63 & 12.30 & 12.30 \\
$\mathrm{GDR}_{2}$ & $1^{-}$ & 16.66 & 17 & 16.61 & 16.60 \\
$2^{+}$ & $2^{+}$ & 5.54 & 15 & 5.18 & 5.14 \\
$\mathrm{ISGQR}$ & $2^{+}$ & 11.60 & 76 & 11.59 & 11.55 \\
$\mathrm{IVGQR}^{+}$ & $2^{+}$ & 21.81 & 45 & 21.69 & 21.68 \\
$3^{-}$ & $3^{-}$ & 3.46 & 21 & 3.21 & 3.19 \\
HEOR & $3^{-}$ & 21.30 & 37 & 21.19 & 21.20 \\
\hline \hline
\end{tabular}

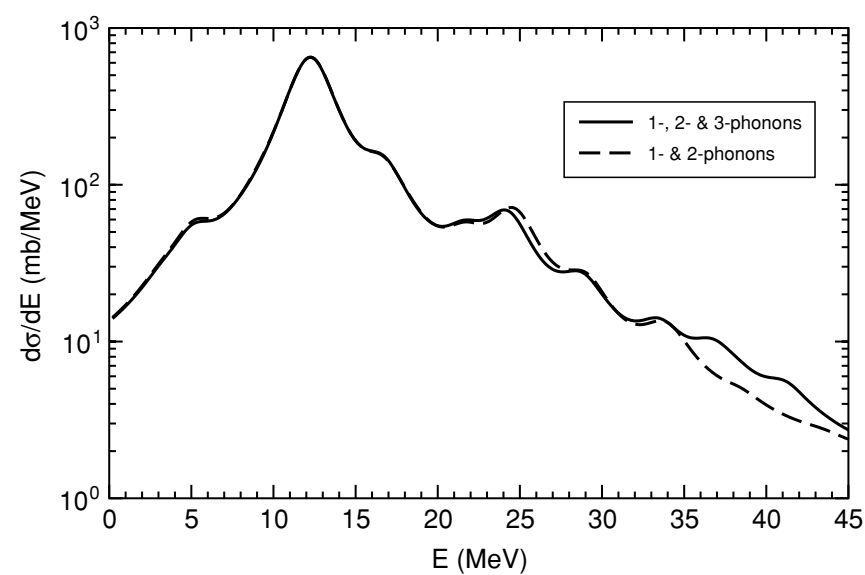

FIG. 5. Comparison of the inelastic cross section for ${ }^{208} \mathrm{~Pb}+{ }^{208} \mathrm{~Pb}$ at $641 \mathrm{MeV} /$ nucleon computed in Ref. [15], including only one- and two-phonon states (dashed line), and the complete calculation going up to three phonons (solid).

is visible. This reduction can be related to the feeding of the three phonon region, possible in the new calculations.

In the high-energy region the contribution of the threephonon states appears to be important. This is confirmed by the decomposition of the inelastic cross section into the one-, two- and three-phonon components as shown in Fig. 6. At this relativistic energies and with such heavy charged ions the one-phonon cross section is clearly dominated by the GDR. In the figure, the small shoulder at $17 \mathrm{MeV}$ is due to the high-lying component of the GDR carrying a small fraction of the dipole strength. The one-phonon component around $22 \mathrm{MeV}$ is the isovector quadrupole vibration. The double phonon component is clearly dominated by the double GDR excitation. In fact from 23 to $34 \mathrm{MeV}$ the inelastic cross section appears to be mainly due to two-phonon states. Indeed, the first peak in this energy region corresponds to the $L=2$ component of $\left|\mathrm{GDR}_{1} \times \mathrm{GDR}_{1}\right\rangle$, the second peak is the $L=2$ component of $\left|\mathrm{GDR}_{1} \times \mathrm{GDR}_{2}\right\rangle$ and the third one is the $L=3$ component of $\left|\mathrm{GDR}_{1} \times \mathrm{IVGQR}\right\rangle(\mathrm{IVGQR}=$ isovector giant

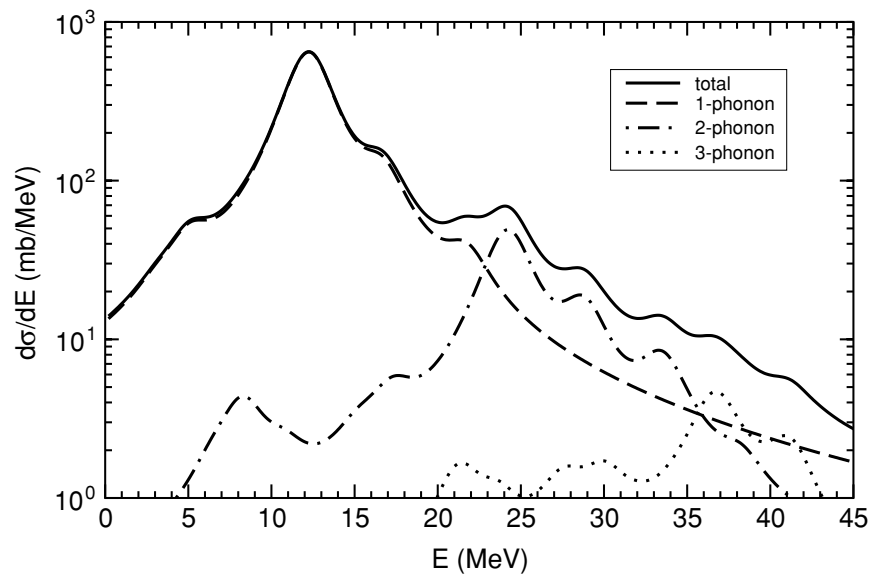

FIG. 6. Decomposition of the inelastic cross section for ${ }^{208} \mathrm{~Pb}+{ }^{208} \mathrm{~Pb}$ at $641 \mathrm{MeV} /$ nucleon (solid line) into the one- (dashed), two- (dot-dashed), and three-phonon components (dotted). 
TABLE VI. Integrated cross section (in $\mathrm{mb}$ ) for ${ }^{208} \mathrm{~Pb}+{ }^{208} \mathrm{~Pb}$ at $641 \mathrm{MeV} /$ nucleon in different energy bins corresponding respectively to the GDR, the two-phonon and the three-phonon regions. In the second row the calculations done with only one- and two-phonon states. In parenthesis the values corresponding to the single, double, and triple GDR states.

\begin{tabular}{lccc}
\hline \hline & $(8-19 \mathrm{MeV})$ & $(22-35 \mathrm{MeV})$ & $(35-45 \mathrm{MeV})$ \\
\hline Three phonons & $3451.2(3078.4)$ & $325.6(227.1)$ & $39.2(18.7)$ \\
Two phonons & $3510.3(3103.3)$ & $348.6(245.0)$ & $4.0(-)$ \\
\hline \hline
\end{tabular}

quadrupole resonance). The double $\mathrm{GDR}_{2}$ state has a small cross section and it cannot be appreciated in the figure. Above $35 \mathrm{MeV}$ the three-phonon modes provide the most important contribution to the spectrum. Indeed, the main peak is due to the triple $\mathrm{DGR}_{1}$, whereas the second one corresponds to the $\left|\mathrm{GDR}_{1} \times \mathrm{GDR}_{1} \times \mathrm{GDR}_{2}\right\rangle$ state.

From Table VI one can see that the integrated cross section for the excitation of the GDR is large at such a relativistic energy, reaching $3.5 \mathrm{~b}$. Then a factor 10 has to be paid each time a new phonon is excited still leaving some sizable cross section for two and three phonon excitations. In the second row we show the results for the calculations done with only one- and two-phonon states. In the three-phonon region we gain a factor 10 when we introduce in the calculation the three-phonon states.

To get a deeper insight in the excitation process, it is interesting to decompose the computed inelastic spectrum in various multipolarities. Let us first start with the dipole strength that strongly dominates at relativistic energies (see Fig. 7). The GDR, which is splitted into a main component at 12.5 $\mathrm{MeV}$ and a smaller peak around $17 \mathrm{MeV}$, is of course the main contributor but one can observe above $35 \mathrm{MeV}$ a small contribution of the three-GDR state coupled to spin and parity $1^{-}$. The quadrupole strength is more complex. Starting at low energy one observes the low-lying collective $2^{+}$state followed by the isoscalar GQR just above $10 \mathrm{MeV}$.

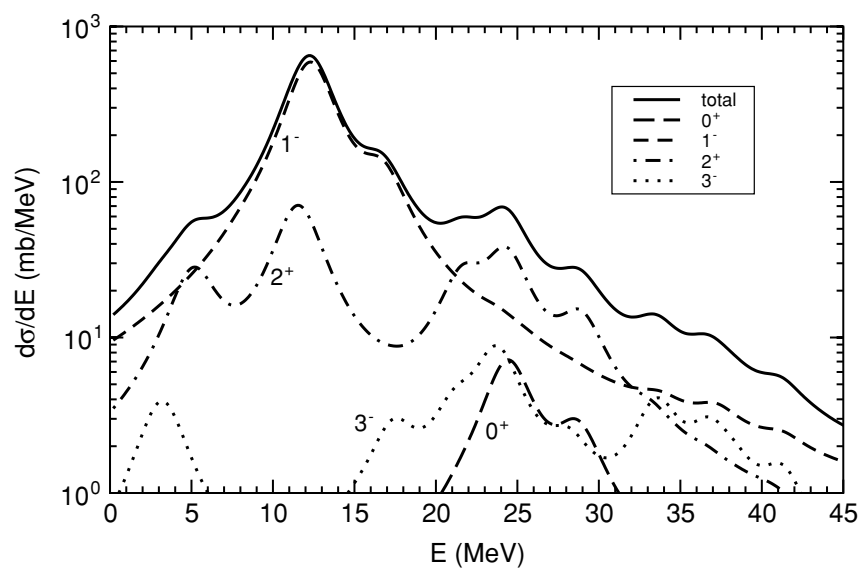

FIG. 7. Decomposition of the inelastic cross section for ${ }^{208} \mathrm{~Pb}+{ }^{208} \mathrm{~Pb}$ at $641 \mathrm{MeV} /$ nucleon (solid line) into different angular momenta, $L=0$ (long-dashed), $L=1$ (short-dashed), $L=2$ (dotdashed), and $L=3$ (dotted).
Except for the small shoulder at $22 \mathrm{MeV}$ coming from the isovector GQR, the strong bump at $25 \mathrm{MeV}$ can be essentially attributed to the double GDR coupled to $2^{+}$. This peak can be directly compared with the monopole strength that corresponds entirely to the double GDR coupled to $0^{+}$. Coming back to the quadrupole response one notices that the two-phonon contribution appears as strong as the one-phonon excitation. A multipole analysis can thus be an interesting way to experimentally isolate the multiphonon contribution. Finally, the octupole response presents both a $3^{-}$and HEOR components around 3 and $24 \mathrm{MeV}$, followed by the triple GDR state coupled to $3^{-}$around $35 \mathrm{MeV}$. This three-phonon component corresponds to the structures observed in the $1^{-}$ response that is nothing but the low spin member of the three-phonon multiplet.

\section{CONCLUSION}

In this article we present, for the first time, microscopic calculations of inelastic cross sections for the triple excitation of giant resonances induced by heavy-ion probes.

We use a microscopic approach based on RPA: the mixing of three-phonon states among themselves and with two- and one-phonon states is considered within a boson expansion approach with Pauli corrections. This is equivalent to introduce anharmonicities corrections to the standard harmonic approximations. At the same time we have also introduced nonlinearities in the external field.

The calculations were done by solving semiclassical coupled-channels equations, the channels being superpositions of multiphonon states. In previous calculations we have considered only one- and two-phonon states obtaining a good agreement with the experimental cross section.

In this article we extend these microscopic calculations by including the three-phonon states. By diagonalizing a quartic microscopic Hamiltonian in the space of up to threephonon states one realizes that a correct description of twophonon states requires the inclusion of one- and three-phonon components. The anharmonicity in most of the cases is of the order of $1 \mathrm{MeV}$. Calculations of the inelastic cross section for the excitation of one-, two-, and three-phonons states have been performed in the framework of this model. The cross section in the DGR energy region is only slightly modified. Thus the previously published results are confirmed. On the contrary, as one could expect, the contribution in the TGR energy region is quite large giving a better agreement with the experimental data. We have also performed calculations in the space of up to four-phonon states. Although the inclusion of the four-phonon states is very important in the wave functions and energies of the three-phonon states, giving rise to a much stronger anharmonicity, their influence on the dynamics is very small.

The decomposition of the inelastic cross section into one-, two-, and three-phonon components shows the importance of the three contributions in different region of the excitation energy. In the case of ${ }^{208} \mathrm{~Pb}+{ }^{208} \mathrm{~Pb}$ at $E / A=641 \mathrm{MeV}$ the separation in energy is very clear and one can distinguish the three region of interest; in the ${ }^{40} \mathrm{Ca}+{ }^{40} \mathrm{Ca}$ at $50 \mathrm{MeV} /$ nucleon 
case the overlap is stronger. In both cases we get an increase in the triple phonon energy region showing once again the importance of the anharmonicity in the internal Hamiltonian and the nonlinearity in the external field.
[1] M. N. Harakeh and A. van der Woude, Giant Resonances: Fundamental High-Frequency Modes of Nuclear Excitation (Clarendon Press, Oxford, 2001).

[2] H. Hemling, Prog. Part. Nucl. Phys. 33, 729 (1994); Ph. Chomaz and N. Frascaria, Phys. Rep. 252, 275 (1995); T. Aumann, P. F. Bortignon, and H. Emling, Annu. Rev. Nucl. Part. Sci. 48, 351 (1998); C. A. Bertulani and V. Yu. Ponomarev, Phys. Rep. 321, 139 (1999).

[3] J. A. Scarpaci et al., Phys. Rev. C 56, 3187 (1997); J. A. Scarpaci et al., Phys. Rev. Lett. 71, 3766 (1993).

[4] N. Frascaria, Nucl. Phys. A687, 154c (2001).

[5] J. A. Scarpaci, Nucl. Phys. A731, 175 (2004).

[6] S. Mordechai et al., Phys. Rev. Lett. 60, 408 (1988); S. Mordechai and C. F. Moore, Nature 352, 393 (1991).

[7] R. Schmidt et al., Phys. Rev. Lett. 70, 1767 (1993); K. Boretzky et al., Phys. Rev. C 68, 024317 (2003).

[8] S. Ilievsky et al., Phys. Rev. Lett. 92, 112502 (2004).

[9] V. Y. Ponomarev et al., Phys. Rev. Lett. 72, 1168 (1994); V. Y. Ponomarev et al., Z. Phys. A 356, 251 (1996); V. Y. Ponomarev et al., Phys. Rev. Lett. 85, 1400 (2000).

[10] D. Brink, Ph.D. thesis, Oxford University, 1955 (unpublished); P. Axel, Phys. Rev. 126, 671 (1962).
[11] B. V. Carlson et al., Phys. Rev. C 60, 014604 (1999).

[12] J. Z. Gu and H. A Weidenmüller, Nucl. Phys. A690, 382 (2001).

[13] M. S. Hussein, B. V. Carlson, L. F. Canto, and A. F. R. de Toledo Piza, Phys. Rev. C 66, 034615 (2002).

[14] C. Volpe, F. Catara, Ph. Chomaz, M. V. Andrés, and E. G. Lanza, Nucl. Phys. A589, 521 (1995); Nucl. Phys. A599, 347c (1996).

[15] E. G. Lanza, M. V. Andrés, F. Catara, Ph. Chomaz, and C. Volpe, Nucl. Phys. A613, 445 (1997); Nucl. Phys. A654, 792c (1999).

[16] M. V. Andrés, F. Catara, E. G. Lanza, Ph. Chomaz, M. Fallot, and J. A. Scarpaci, Phys. Rev. C 65, 014608 (2001).

[17] M. Fallot, Ph. Chomaz, M. V. Andrés, F. Catara, E. G. Lanza, and J. A. Scarpaci, Nucl. Phys. A729, 699 (2003).

[18] M. Hage-Hassan and M. Lambert, Nucl. Phys. A188, 545 (1972).

[19] C. Volpe, Ph. Chomaz, M. V. Andrés, F. Catara, and E. G. Lanza, Nucl. Phys. A647, 246 (1999).

[20] E. G. Lanza, M. V. Andrés, F. Catara, Ph. Chomaz, and C. Volpe, Nucl. Phys. A636, 452 (1998).

[21] M. Fallot, Ph.D. thesis, IPNO2002T-02-05; http://tel.archivesouvertes.fr/tel-00002925/en/ 